\title{
Associação entre a presença de anemia ferropriva com variáveis socioeconômicas e rendimento escolar
}

\author{
Association between the occurence of iron-deficiency anemia with socioeconomic \\ variables and school performance
}

Pâmela Alves Silva ${ }^{1}$, Thaysa Martins Justino ${ }^{1}$, Roberta Angélica Da Silva Heitor ${ }^{1}$, Felipe Florentino dos Santos ${ }^{2}$, Amanda Rodrigues Barbosa ${ }^{1}$, Bruna Gabriela Rocha ${ }^{3}$, Ana Carolliny Fernandes Faria ${ }^{1}$, Débora Araújo Silva ${ }^{1}$, Letícia Gonçalves Resende Ferreira ${ }^{4}$, Wander Valadares de Oliveira Júnior ${ }^{4}$, Melina de Barros Pinheiro Inácio ${ }^{5}$, Danyelle Romana Alves Rios ${ }^{5}$, Caroline Pereira Domingueti ${ }^{5}$

\begin{abstract}
RESUMO
Modelo do estudo: Observacional transversal.

Objetivo: Avaliar a associação entre a presença de anemia ferropriva com variáveis socioeconômicas e rendimento escolar.

Método: Foram incluídas no estudo 124 crianças com idade entre seis e oito anos, estudantes do ensino fundamental de escolas municipais, as quais foram divididas em dois grupos de acordo com a presença $(n=32)$ ou ausência de anemia $(n=92)$. Os níveis de hemoglobina e ferro sérico foram determinados por método colorimétrico, a contagem de hemácias foi realizada utilizando a câmara de Neubauer, o hematócrito foi avaliado utilizando centrífuga de microhematócrito, e foram calculados os índices hematimétricos volume corpuscular médio, hemoglobina corpuscular média e concentração de hemoglobina corpuscular média. O desempenho escolar das crianças foi fornecido pelas escolas participantes e as variáveis socioeconômicas foram obtidas através de preenchimento de ficha clínica e do questionário socioeconômico da Associação Brasileira das Empresas de Pesquisa pelos pais ou responsáveis.

Resultados: A prevalência de anemia ferropriva nos escolares foi de $25,8 \%$ que é considerada pelos parâmetros da OMS uma prevalência moderada. Foi observada uma maior proporção de crianças sem anemia que apresentaram melhores conceitos escolares e que pertencem aos níveis socioeconômicos mais altos do que de crianças com anemia. Contudo, não foram observadas diferenças estatisticamente significativas entre os grupos com relação ao rendimento escolar e as variáveis socioeconômicas.
\end{abstract}

Conclusão: Uma prevalência moderada de anemia ferropriva foi encontrada nas crianças com idade entre seis e oitos anos, entretanto, não foi observada uma associação significativa entre a anemia ferropriva com variáveis socioeconômicas e o rendimento escolar.

Palavras-chaves: Anemia Ferropriva. Desenvolvimento Infantil. Classe Social.

1. Graduada em Farmácia.

2. Graduando em Farmácia.

3. Mestranda em Ciências Farmacêuticas.

4. Doutoranda em Ciências da Saúde.

5. Doutora em Ciências Farmacêuticas.

Universidade Federal de São João del-Rei - Campus

Centro Oeste Dona Lindu - Divinópolis - MG - Brasil
CORRESPONDÊNCIA: Caroline Pereira Domingueti caroldomingueti@ufsj.edu.br Universidade Federal de São João Del-Rei Campus Centro-Oeste Dona Lindu Rua Sebastião Gonçalves Coelho, 400 - Chanadour Cep: 35501-296 - Divinópolis - MG - Brasil

Recebido em 05/04/2018 Aprovado em 24/10/2018 


\begin{abstract}
Study design: Cross-sectional observational.

Objective: Evaluate the association between the occurence of iron-deficiency anemia with socioeconomic variables and school performance.

Method: They were included in the study 124 children aged between six and eight years old, municipal elementary school students, which were divided in two groups according to the presence $(n=32)$ or absence of anemia $(n=92)$. Hemoglobin and serum iron levels were determined by colorimetric method, red blood cells count was performed using Neubauer chamber, hematocrit was evaluated using microhematocrit centrifuge, and mean corpuscular volume, mean corpuscular hemoglobin, and mean corpuscular hemoglobin concentration hematimetric indexes were calculated. The school performance of children was provided by participating schools and socioeconomic variables were obtained by filling out the clinic file and the socioeconomic questionnaire of Brazilian Association of Research Companies by parents or guardians.

Results: The prevalence of iron-deficiency anemia in school children was $25.8 \%$, which is considered to be moderate. The proportion of better school grades was higher in children without anemia and in those belonging to the upper socioeconomic levels. However, it was not observed statistically differences between groups regarding school performance and socioeconomic variables.

Conclusion: A moderate prevalence of iron-deficiency anemia was found in children aged between six and eight years old, however, it was not observed a significant association between irondeficiency anemia with socioeconomic variables and school performance.
\end{abstract}

Keywords: Anemia, Iron-Deficiency. Child Development. Social Class

\section{INTRODUÇÃO}

A anemia é definida pela Organização Mundial da Saúde $(\mathrm{OMS})^{1}$ como a condição na qual a concentração sanguínea de hemoglobina está abaixo dos valores considerados normais para a idade, sexo, estado fisiológico e altura. Estima-se que cerca de 2,15 bilhões de pessoas, quase $40 \%$ da população mundial, apresentam carência de ferro ou níveis baixos de hemoglobina ${ }^{2,3}$. A anemia ferropriva consiste na principal causa de anemia, contribuindo para aproximadamente $42 \%$ dos casos em crianças com menos de cinco anos de idade 4 . Ela é caracterizada pela diminuição das reservas de ferro e da saturação da transferrina, níveis reduzidos de ferro sérico e de hemoglobina, diminuição do hematócrito, microcitose e hipocromia ${ }^{5,6}$.

Vários fatores têm sido associados com a presença de anemia ferropriva em crianças, tais como a prematuridade, o baixo peso ao nascimento, hipóxia crônica, infecções frequentes, baixo nível socioeconômico, baixa ingestão de carne ou de vitamina $C$, menor diversidade alimentar, curto tempo de aleitamento materno, falta de cuidados da mãe com o filho, parasitoses intestinais, baixa escolaridade materna, falta de acesso aos serviços de saúde pública, dentre outros3,7. Na infância, devido ao aumento da demanda do mineral, a deficiência de ferro pode gerar prejuízos no crescimento e no desenvolvimento do sistema nervoso central, os quais podem ser irreversíveis ${ }^{4,8}$.

Crianças anêmicas podem apresentar um comprometimento do desenvolvimento cognitivo e neuropsicomotor em decorrência da menor produção de hemoglobina, e consequente menor transporte de oxigênio para o cérebro, o que pode alterar a neurotransmissão e a mielinização ${ }^{9}$. A deficiência de ferro provoca ainda alterações na estrutura dos dendritos das células piramidais do hipocampo, comprometendo a memória ${ }^{10}$. A anemia ferropriva foi ainda associada a uma menor capacidade de aprendizado e a um pior desempenho no teste de Quociente de Inteligência ${ }^{8}$.

A avaliação da associação entre a anemia ferropriva e o desempenho escolar de crianças em idade escolar, e dos fatores socioeconômicos que estão associados com a presença da anemia ferropriva na infância é extremamente importante, já que pode direcionar a adoção de medidas públicas de saúde que visem a prevenção e o tra- 
tamento da anemia ferropriva nas crianças desta faixa etária, contribuindo assim para uma melhoria da capacidade de aprendizagem e do rendimento escolar.

Portanto, este trabalho teve como objetivo avaliar a associação entre a presença de anemia ferropriva e variáveis socioeconômicas e o rendimento escolar em crianças com idade entre seis e oito anos.

\section{MATERIAIS E MÉTODOS}

O município de Divinópolis/MG possui 33 escolas municipais, as quais apresentam em torno de 2000 alunos cursando o primeiro e o segundo anos do ensino fundamental. O cálculo amostral foi realizado utilizando como prevalência de anemia ferropriva estimada em $26,9 \%^{11}$, com precisão de $5 \%$ e intervalo de confiança de 95\%, resultando em 119 crianças. A esse valor acrescentou-se $10 \%$ para compensar possíveis perdas, resultando em um total de 131 crianças.

Oito escolas municipais foram selecionadas para participar deste estudo observacional transversal de forma aleatória. Todos os estudantes do primeiro e do segundo anos do ensino fundamental destas oito escolas municipais, e os responsáveis por estes, foram convidados a participar do estudo, e 124 aceitaram o convite. Assim, foram incluídas no estudo 124 crianças com idade entre seis e oito anos. As crianças foram divididas em dois grupos de acordo com a presença ou ausência de anemia ferropriva, a qual foi definida por níveis de hemoglobina inferiores a $11,5 \mathrm{~g} / \mathrm{dL} 1$. Foi excluída a presença de outras causas de anemia, que não a ferropriva, por meio da não inclusão no estudo de crianças que apresentam talassemia, anemia falciforme, doença renal crônica, dentre outras causas comuns de anemia na infância.

Foram coletadas de cada criança participante do estudo, amostras de $4,0 \mathrm{~mL}$ de sangue venoso em tubos com anticoagulante EDTA e sem anticoagulante para obtenção do soro. As amostras de sangue total foram utilizadas para a contagem de hemácias, dosagem da hemoglobina e determinação do hematócrito. O soro foi utilizado para a determinação dos níveis séricos de ferro.

A contagem de hemácias foi feita manualmente utilizando microscópio e câmara de
Neubauer. Os níveis de hemoglobina e de ferro sérico foram determinados por método colorimétrico utilizando os kits Hemoglobina (Bioclin $\circledast$ ) e Ferro Sérico (Bioclin ${ }^{\circledR}$ ). A determinação do hematócrito foi realizada utilizando tubos capilares e a centrífuga de microhematócrito. Os índices hematimétricos volume corpuscular médio (VCM), hemoglobina corpuscular média (HCM), concentração de hemoglobina corpuscular média ( $\mathrm{CHCM}$ ) foram calculados ${ }^{12}$.

Dados clínicos e socioeconômicos foram obtidos por meio de entrevista com os responsáveis pelas crianças e preenchimento de uma ficha clínica. O nível socioeconômico das famílias foi obtido por meio de preenchimento do questionário socioeconômico da Associação Brasileira de Empresas de Pesquisa ${ }^{13}$, o qual se baseia na quantidade de itens que a família possui, tais como automóvel, geladeira, dentre outros, e na escolaridade do chefe de família. O nível socioeconômico foi classificado em A1, A2, B1, B2, C1, C2, D e E13.

$O$ rendimento escolar das crianças em língua portuguesa, matemática, ciências biológicas e ciências humanas (geografia e história) foi fornecido pelas diretoras das escolas municipais. 0 rendimento escolar foi classificado em conceitos: A (ótimo), B (muito bom), C (bom), D (regular).

Os resultados obtidos foram analisados utilizando o programa estatístico SPSS 20.0. Foi realizado o teste de normalidade Shapiro-Wilk para as variáveis contínuas. Para as variáveis que apresentaram distribuição normal, foram calculados os valores de média e desvio-padrão, e foi empregado o teste $\mathrm{T}$ de Student para comparação entre os grupos. Para as variáveis que não apresentaram distribuição normal, foram calculadas a mediana e os percentis $25 \%$ e $75 \%$, e foi empregado o teste Mann Whitney U para comparação entre os grupos. As variáveis categóricas foram apresentadas como frequências absolutas e relativas, e foi empregado o teste qui-quadrado para comparação entre estas variáveis. Foi considerado significativo o valor $p<0,05$.

Esse estudo foi aprovado pelo Comitê de Ética em Pesquisa da Universidade Federal de São J oão Del Rei (CAAE: 38854914.8.0000.5545) e foi realizado de acordo com as recomendações da Declaração de Helsinki. Todos os responsáveis pelos participantes receberam esclarecimento 
sobre os objetivos do projeto e assinaram o Termo de Consentimento Livre e Esclarecido, e todos os escolares selecionados assinaram o Termo de Assentimento Livre e Esclarecido.

\section{RESULTADOS}

Dentre as 124 crianças incluídas no estudo, 32 apresentavam anemia, indicando uma pre- valência de $25,8 \%$. As características clínicas, socioeconômicas e laboratoriais das crianças agrupadas de acordo com a presença ou ausência de anemia estão apresentadas na Tabela 1 .

Os níveis de hemoglobina ( $p<0,001$ ), hematócrito $(p=0,001)$ e ferro sérico $(p=0,019)$, e os índices hematimétricos HCM $(p<0,001)$ e CHCM $(p<0,001)$ foram significativamente menores nas crianças com anemia em comparação com aquelas

Tabela 1

Características clínicas, socioeconômicas e laboratoriais das crianças com e sem anemia

\begin{tabular}{|c|c|c|c|}
\hline & Crianças sem anemia & Crianças com anemia & Valor $\mathrm{p}$ \\
\hline Número de indivíduos (n) & 92 & 32 & \\
\hline Sexo masculino $(n, \%)$ & $50(54,3)$ & $16(50,0)$ & NS \\
\hline Idade (meses) & $81(72-84)$ & $76(72-84)$ & NS \\
\hline Peso $(\mathrm{Kg})$ & $23,4(21,2-27,4)$ & $23,2(21,2-25,7)$ & NS \\
\hline Peso ao nascimento $(\mathrm{g})$ & $3049 \pm 640$ & $3297 \pm 518$ & NS \\
\hline Hemoglobina ( $g / d L)$ & $13,5 \pm 1,1$ & $10,4 \pm 0,7 *$ & $<0,001$ \\
\hline Hematócrito (\%) & $37(36-38)$ & $36(34-37)^{*}$ & 0,001 \\
\hline Número de hemácias (hemácias/mm³) & $4309879 \pm 737802$ & $4318064 \pm 1184684$ & NS \\
\hline $\operatorname{VCM}(\mathrm{mm} 3)$ & $84(74-92)$ & $83(66-115)$ & NS \\
\hline HCM (pg) & $31(27-35)$ & $25(20-29) *$ & $<0,001$ \\
\hline CHCM $(g / d L)$ & $36 \pm 2$ & $29 \pm 3^{*}$ & $<0,001$ \\
\hline Ferro $(\mu \mathrm{g} / \mathrm{dL})$ & $59 \pm 27$ & $47 \pm 22 *$ & 0,019 \\
\hline Dificuldade de aprendizado $(n, \%)$ & $13(14,1)$ & $6(18,8)$ & NS \\
\hline Capacidade de ler/escrever $(n, \%)$ & $48(61,5)$ & $15(65,2 \%)$ & NS \\
\hline Renda familiar mensal $(\mathrm{R} \$)$ & $\begin{array}{c}1600,00 \\
(900,00-2800,00)\end{array}$ & $\begin{array}{c}1500,00 \\
(1000,00-2200,00)\end{array}$ & N S \\
\hline 1 a 4 pessoas dependentes da renda $(n, \%)$ & $61(76,2)$ & $20(76,9)$ & NS \\
\hline 5 a 7 pessoas dependentes da renda $(n, \%)$ & $19(23,8)$ & $6(23,1)$ & NS \\
\hline Renda familiar por pessoa $(\mathrm{R} \$$ ) & $\begin{array}{c}442,00 \\
(267,00-646,00)\end{array}$ & $\begin{array}{c}400,00 \\
(237,00-600,00)\end{array}$ & $\mathrm{NS}$ \\
\hline Pai trabalha fora $(n, \%)$ & $66(95,7)$ & $21(100,0)$ & NS \\
\hline Mãe trabalha fora $(n, \%)$ & $51(60,0)$ & $12(46,2)$ & NS \\
\hline 1 ou 2 filhos $(n, \%)$ & $61(72,6)$ & $18(69,2)$ & NS \\
\hline 3 a 5 filhos $(n, \%)$ & $23(27,4)$ & $8(30,8)$ & NS \\
\hline Criança é primeiro ou segundo filho $(n, \%)$ & $54(83,1)$ & $16(88,9)$ & NS \\
\hline Criança é terceiro, quarto ou quinto filho $(n, \%)$ & $11(16,9)$ & $2(11,1)$ & NS \\
\hline Tempo de aleitamento (meses) & $7(3-13)$ & $12(6-17)$ & NS \\
\hline Mãe realizou o pré-natal durante a gestação $(n, \%)$ & $86(100,0)$ & $28(100,0)$ & NS \\
\hline Mãe tomou sulfato ferroso durante gestação $(n, \%)$ & $71(83,5)$ & $26(96,3)$ & NS \\
\hline
\end{tabular}

$\mathrm{VCM}=$ volume corpuscular médio, $\mathrm{HCM}=$ hemoglobina corpuscular média, $\mathrm{CHCM}=$ concentração de hemoglobina corpuscular média . Variáveis que apresentaram distribuição normal foram expressas como média \pm desvio padrão e foram comparadas por meio do teste T de Student. Variáveis que não apresentaram distribuição normal foram expressas como mediana (percentil 25\% - 75\%) e foram comparadas pelo Teste Mann-Whitney U. Variáveis categóricas foram expressas como frequência absoluta e relativa $\mathrm{n}$ (\%) e foram comparadas por meio do teste do Qui-Quadrado $\left(\mathrm{c}^{2}\right)$. ${ }^{\star} \mathrm{p}<0,05$ para crianças com anemia em comparação com crianças sem anemia. NS = não significativo. 
sem anemia. Não foram observadas diferenças significativas entre as crianças com e sem anemia com relação ao sexo, idade, peso, peso ao nascimento, dificuldade de aprendizado, capacidade de ler e escrever, número de hemácias, índice hematimétrico VCM, renda familiar mensal, número de pessoas dependentes da renda, renda familiar por pessoa, proporção de pais e mães que trabaIham fora, número de filhos, ordem de nascimento da criança, tempo de aleitamento materno, realização de pré-natal durante a gestação, administração de sulfato ferroso durante a gestação.

Os rendimentos escolares em língua portuguesa, matemática, ciências biológicas e ciências humanas das crianças com e sem anemia estão apresentados na Figura 1. A frequência de crianças sem anemia que tiveram conceito $A$ em língua portuguesa, ciências biológicas e ciências humanas foi maior do que a proporção de crianças com anemia que tiveram conceito $A$ nestas matérias. Por outro lado, as frequências de crianças sem anemia que tiveram conceito $B$ em língua portuguesa, ciências biológicas e ciências humanas, e que tiveram conceito $\mathrm{C}$ em língua portuguesa e matemática foram menores do que as proporções de crianças com anemia que tiveram conceitos $B$ e $C$ nestas matérias. Contudo, não foram observadas diferenças significativas entre as crianças com e sem anemia para o rendimento escolar nestas disciplinas.

O nível socioeconômico das famílias das crianças que possuem ou não anemia, quem é o chefe da família, a escolaridade do chefe da família e a escolaridade da mãe estão apresentados na Figura 2. A maioria das famílias em que as crianças não possuem anemia pertencem ao nível B2 $(32,5 \%)$, seguido por C1 $(28,7 \%)$, C2 $(22,5 \%)$, D $(11,2 \%)$ e B1 $(3,9 \%)$. Já entre as famílias das crianças que apresentam anemia, a maior parte destas é classificada como C1 $(40,9 \%)$, seguido por C2 e D na mesma proporção $(22,7 \%)$ e B2 $(13,6 \%)$. Apesar de não terem sido observadas diferenças significativas entre os grupos com relação ao nível socioeconômico, a proporção de famílias de crianças sem anemia pertencentes aos níveis socioeconômicos mais altos (B1 e B2) foi maior e daquelas pertencentes ao nível socioeconômico mais baixo (D) foi menor em comparação com as famílias de crianças com anemia.
Não foram observadas diferenças significativas entre as crianças com e sem anemia com relação a quem é o chefe da família. Contudo, foi observada uma diferença significativa entre os grupos com relação à escolaridade dos chefes de família. Nas famílias de crianças com anemia, a proporção de chefes de família que completou o ensino fundamental foi maior, enquanto que a proporção que completou o ensino médio ou a 4 a série do ensino fundamental foi menor, em comparação com as famílias de crianças sem anemia. Não foram observadas diferenças significativas entre os grupos com relação ao nível de escolaridade materna. Contudo, a proporção de mães de crianças com anemia que é analfabeta ou estudou até a 3a série do ensino fundamental foi maior, enquanto que a proporção que completou o ensino superior foi menor em comparação com as mães de crianças com anemia.

\section{DISCUSSÃO}

A anemia ferropriva atinge aproximadamente 2 bilhões de pessoas em todo mundo, atingindo mais de $50 \%$ das crianças entre seis meses e cinco anos de idade, constituindo em um importante problema de saúde pública ${ }^{8}$. No Brasil, uma revisão sistemática demonstrou que a prevalência de anemia em crianças com menos de cinco anos de idade é de $53 \%$, indicando uma alta prevalência, principalmente nas crianças menores de dois anos ${ }^{14}$.

No presente estudo foi encontrada uma prevalência de anemia ferropriva de $25,8 \%$ nos escolares com idade entre seis e oito anos, sendo considerada pelos parâmetros da OMS uma prevalência moderada1. Outros estudos realizados em diferentes regiões do Brasil também encontraram uma prevalência moderada de anemia em crianças com idade igual ou superior a seis $\operatorname{anos}^{11,15,16,17 .}$

De modo semelhante a outros estudos epidemiológicos ${ }^{11}$, o diagnóstico de anemia ferropriva foi realizado no presente estudo por meio da dosagem de hemoglobina, o qual consiste no exame mais utilizado para o diagnóstico em escala populacional, devido à praticidade ${ }^{1}$. A presença de anemia ferropriva foi definida por níveis de hemoglobina inferiores a $11,5 \mathrm{~g} / \mathrm{dL}$, o qual consiste no 


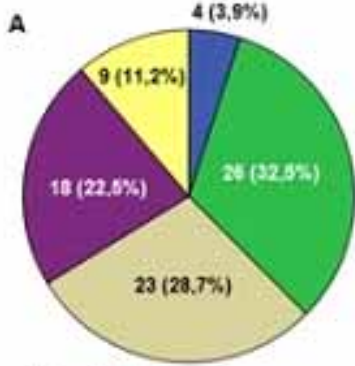

$$
\begin{aligned}
& \text { Familia das crianças } \\
& \text { sem anemia }
\end{aligned}
$$

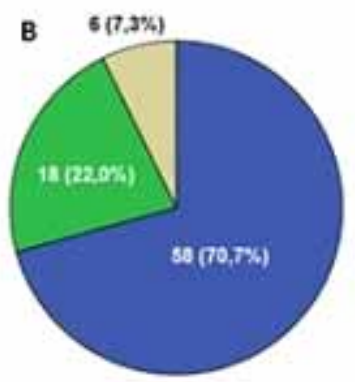

Chefe das familias em que as crianças não possuem anemia

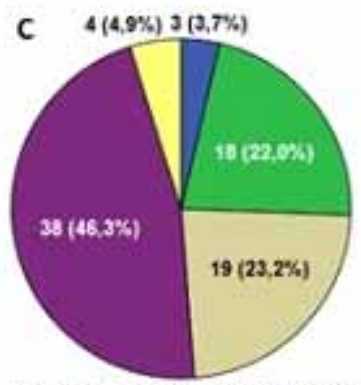

Chefe das familias em que as crianças não possuem anemia

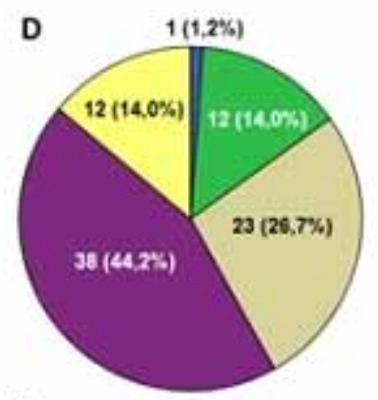

Mães de crianças sem anemia Mảes de crianças com anemia

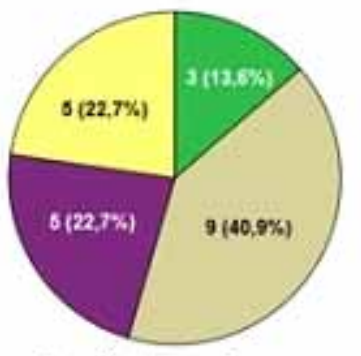

Familia das crianças com anemia

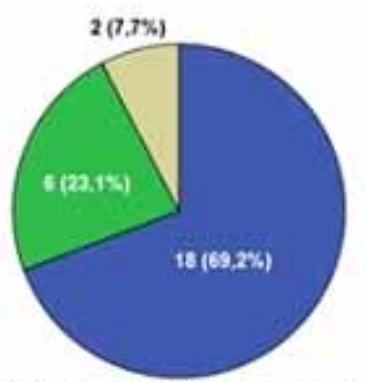

Chefe das familias em que as crianças possuem anemia

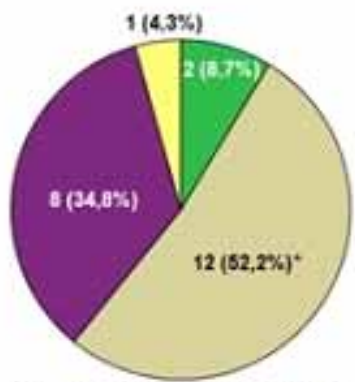

Chefe das familias em que as

crianças possuem anemia

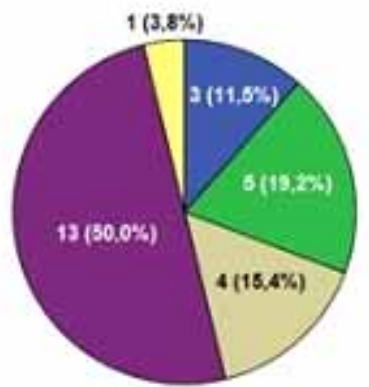

Nivel Socioeconômico

a $\mathrm{B} 1$

B2

$\square \mathrm{C} 1$

a $\mathrm{C} 2$

व D

\section{Chefe de Familia}

u Pai

a Mãe

- Outros

\section{Escolaridade do Chefe da Familia}

- Analfabeto/atẻ 3 série do ensino fundamental

- Completou a 4 série do ensino fundamental

- Ensino fundamental completo

a Ensino médio completo

ㅁ. Ensino superior completo

\section{Escolaridade Materna}

- Analfabeto/até 3 série do ensino fundamental

- Completou a 4 série do ensino fundamental

a Ensino fundamental completo

- Ensino médio completo

․ Ensino superior completo

Figura 1: Rendimento escolar das crianças com e sem anemia em: A - Língua Portuguesa; B - Matemática; C - Ciências Biológicas; D - Ciências Humanas.

Variáveis foram expressas como frequência absoluta e relativa n (\%) e foram comparadas por meio do teste do Qui-Quadrado ( $\left.\chi^{2}\right)$, não tendo sido encontradas diferenças significativas entre os grupos. 

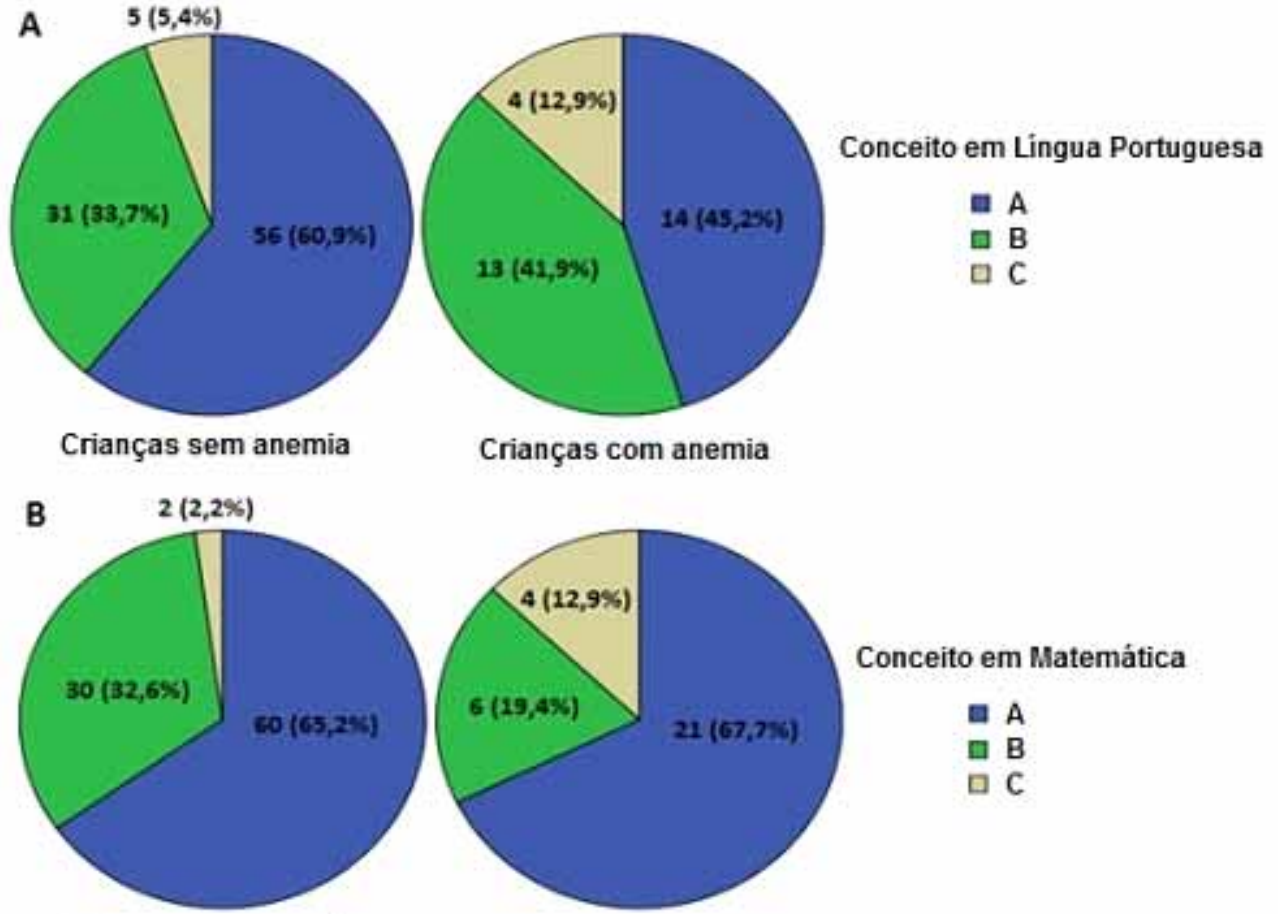

Crianças com anemia

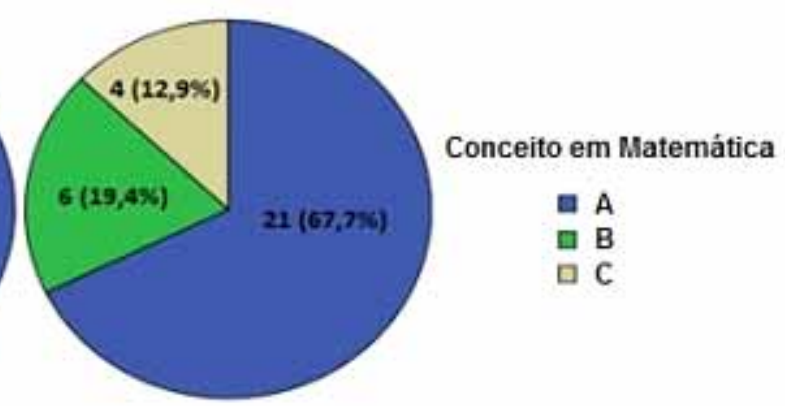

Crianças sem anemia

Crianças com anemia

C

$1(1,1 \%)$

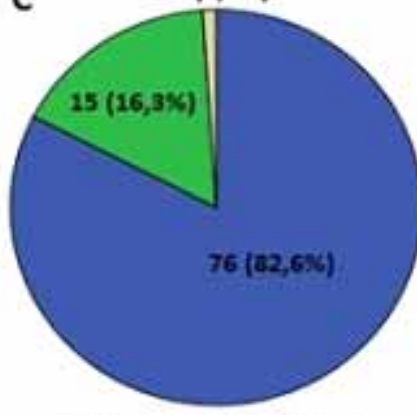

Crianças sem anemia

D

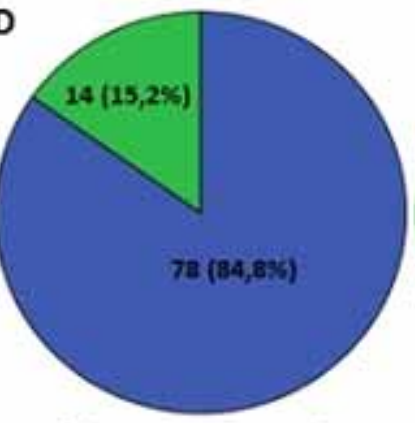

Crianças sem anemia

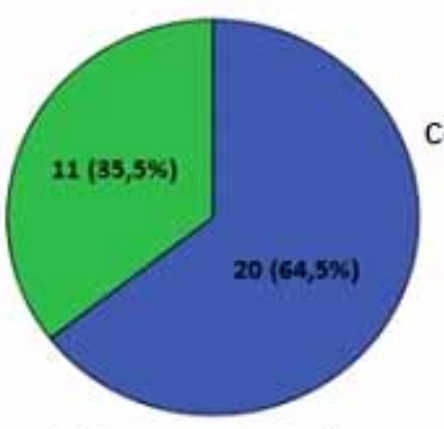

\section{Conceito em Ciências Biológicas}

a $\mathrm{A}$

ㅁ B

므

\section{Crianças com anemia}

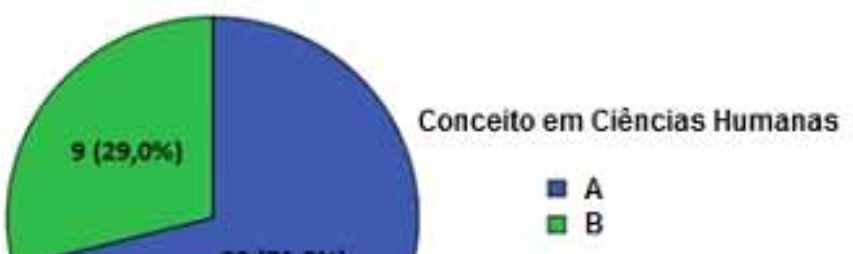

Figura 2: Variáveis socioeconômicas das crianças que possuem ou não anemia: A - Nível socioeconômico da família; B - Chefe da família; C - Escolaridade do chefe da família; D - Escolaridade da mãe.

Variáveis foram expressas como frequência absoluta e relativa $\mathrm{n}(\%)$ e foram comparadas por meio do teste do Qui-Quadrado $\left(\chi^{2}\right)$. ${ }^{*} \mathrm{p}<0,05$ para chefe de família com ensino fundamental completo em comparação com chefe de família das demais escolaridades no grupo em que as crianças possuem anemia. 
valor de referência preconizado pela OMS para crianças com idade entre seis e onze anos ${ }^{1}$.

Apesar dos níveis séricos de ferro terem sido determinados no presente estudo e terem se mostrado significativamente menores no grupo de crianças com anemia, caracterizando a presença de anemia ferropriva, os mesmos não foram utilizados para o estabelecimento do diagnóstico de anemia ferropriva, devido ao fato de que nem sempre os níveis séricos de ferro estão abaixo do valor de referência nesta patologia ${ }^{18}$.

As principais alterações observadas no hemograma de indivíduos com anemia ferropriva são a diminuição dos níveis de hemoglobina, redução do hematócrito, presença de número normal ou diminuído de hemácias, microcitose e hipocromia 5 . Condizente com os achados laboratoriais característicos da anemia ferropriva, os níveis de hematócrito foram significativamente menores nas crianças com anemia em comparação com aquelas sem anemia, e não foram observadas diferenças significativas com relação ao número de hemácias. As crianças anêmicas apresentaram HCM significativamente menor do que as não anêmicas, o que é condizente com a presença de hipocromia decorrente da menor produção de hemoglobina. Apesar de a microcitose ser um achado laboratorial característico da anemia ferropriva, não foram observadas diferenças significativas no VCM entre crianças anêmicas e não anêmicas.

No que diz respeito ao impacto no desempenho escolar, foi observada no presente estudo, uma maior proporção de crianças sem anemia que apresentaram melhor conceito (A) e uma menor proporção de crianças sem anemia que apresentaram piores conceitos ( $\mathrm{B}$ ou $\mathrm{C}$ ), indicando uma maior prevalência de anemia nas crianças que apresentam pior rendimento escolar. Contudo, não foram observadas diferenças estatisticamente significativas entre crianças anêmicas e não anêmicas quanto ao rendimento escolar, o que pode ser decorrente do pequeno tamanho amostral. Além disso, outras variáveis que não a anemia, como por exemplo o baixo peso ao nascer e o baixo nível socioeconômico familiar, podem interferir no desempenho escolar das crianças, podendo interferir nos resultados encontrados.

Um estudo realizado com alunos com idade entre sete e dezessete anos verificou que as cri- anças anêmicas obtiveram um rendimento escolar insuficiente (conceitos D e E) em língua portuguesa e em matemática e um pior desempenho no teste de memória visual quando comparado às crianças não anêmicas ${ }^{19}$. A deficiência de ferro tem sido destacada como o problema nutricional de maior impacto sobre o crescimento, o desenvolvimento psicossocial e o desenvolvimento motor e cognitivo. Crianças anêmicas têm sido consideradas mais sensíveis a prejuízos no desenvolvimento cognitivo e neuropsicomotor devido ao comprometimento de processos fisiológicos relacionados à produção de hemoglobina e consequente transporte de oxigênio ao cérebro, alterando a neurotransmissão e mielinização. A anemia ferropriva em escolares é indiscutivelmente deletéria, pois crianças anêmicas apresentam sonolência durante as atividades e, com isso, a sua atenção fica prejudicada, levando ao baixo rendimento escolar ${ }^{9}$. Uma revisão sistemática e meta-análise ainda demonstrou que a suplementação diária com ferro em crianças com idade entre 5 e 12 anos melhora os escores cognitivos globais, o quociente de inteligência, a atenção e a concentração ${ }^{20}$.

Não foram encontradas diferenças significativas entre os grupos em relação ao nível socioeconômico, no entanto foi observada uma proporção maior de famílias de crianças sem anemia pertencentes aos níveis socioeconômicos mais altos (B1 e B2) e uma proporção menor daquelas pertencentes aos níveis socioeconômicos mais baixos (D) quando em comparação com as famílias de crianças com anemia. No estudo de Santos et al. $(2004)^{21}$, foi observado também uma maior proporção de famílias de crianças com anemia pertencentes ao nível socioeconômico mais baixo. As condições sociais e econômicas das famílias que possuem renda mais baixa podem contribuir para agravar a deficiência de ferro, seja por uma alimentação inadequada, precariedade do saneamento básico, menor acesso a serviços de saúde e a informações para prevenção e tratamento dessa doença22.

A proporção de crianças com anemia tende a ser maior entre aquelas pertencentes às famílias com renda mais baixa, devido à falta de uma alimentação adequada bem como saneamento básico precário. Entretanto, nem sempre esta 
associação é observada, uma vez que um aumento da renda não garante a diminuição da prevalência de anemia, pois esta carência também é encontrada em populações de níveis socioeconômicos altos, em decorrência de maus hábitos alimentares ${ }^{5}$. A associação entre a presença de anemia e a renda familiar não foi encontrada no presente estudo, já que a renda familiar mensal e a renda familiar por pessoa dos grupos com e sem anemia eram próximas. No estudo de Beinner et al. ${ }^{23}$, que foi realizado com meninas adolescentes, também não foi observada uma associação significativa entre a anemia e a renda familiar.

Outra característica socioeconômica importante no desenvolvimento da anemia é a escolaridade dos pais, uma vez que pode gerar maiores chances de emprego e de renda, resultando em melhor acesso a alimentação. No que se refere à escolaridade materna, um nível maior de conhecimento pode influenciar na atenção e saúde da criança ${ }^{2}$. A maior parte dos chefes das famílias das crianças sem anemia concluíram o ensino médio, já entre as crianças com anemia a maior parte dos chefes das famílias possuem ensino fundamental completo. Em relação às mães de crianças sem anemia é possível notar que a proporção que completou o ensino superior é maior e a proporção que é analfabeta ou estudou até a 3a série do ensino fundamental é menor em comparação com as mães de crianças com anemia.

Nos dois grupos de crianças avaliados, a maioria das famílias apresentou até quatro pessoas dependentes da renda familiar mensal, resultado semelhante ao observado por Rocha et al. ${ }^{24}$. A constituição familiar é considerada um fator de risco importante para o desenvolvimento de anemia, uma vez que um número maior de crianças pequenas na residência promove aumento da demanda por alimentos e cuidados, que nem sempre podem ser supridos pela renda da família e disponibilidade dos pais ${ }^{25}$.

O número de filhos apresentou resultados semelhantes nos grupos de crianças com e sem anemia, uma vez que a maior parte das famílias possui um ou dois filhos. Um estudo demonstrou que as crianças possuem dois ou mais irmãos com menos de cinco anos de idade apresentam um maior risco de desenvolverem anemia, pois quanto maior o número de crianças pequenas na família, menor é a atenção, a qual inclui os cuidados com a alimentação e saúde, fornecida pelos pais para cada criança ${ }^{25}$.

Cerca de $60 \%$ das mães do grupo sem anemia e $46,2 \%$ das mães do grupo com anemia trabalham fora de casa, resultados semelhantes aos encontrados por Pessoa et al. ${ }^{26}$. A mãe trabalhar fora de casa pode levar a melhoria na renda familiar, refletindo na aquisição de alimentos e acesso a saúde, o que pode contribuir para uma menor frequência de anemia entre as crianças ${ }^{27}$.

No presente estudo todas as mães realizaram o pré-natal, a maioria tomou sulfato ferroso durante a gestação e o tempo médio de aleitamento foi maior do que seis meses em ambos os grupos de crianças com e sem anemia. A maior atenção a realização do pré-natal, resultando em diminuição do baixo peso ao nascer, da prematuridade e da anemia materna, além do incentivo ao aleitamento materno exclusivo e a orientação sobre a adequada suplementação alimentar são medidas importantes para a redução da prevalência de anemia em crianças $^{28}$.

O estudo apresentou algumas limitações, como tamanho amostral pequeno, desenho transversal e o fato que a avaliação da presença de anemia ferropriva foi feita somente pela dosagem da hemoglobina. Os níveis de hemoglobina são habitualmente usados no diagnóstico desta doença, mas não são tão precisos quanto os níveis de ferritina sérica. No entanto, por questões de praticidade, a dosagem da concentração de hemoglobina é o exame mais utilizado para o diagnóstico da anemia ferropriva em escala populacional $^{27}$.

\section{CONCLUSÃO}

Neste estudo, foi observada uma prevalência moderada de anemia ferropriva nos escolares com idade entre seis e oito anos do município de Divinópolis-MG. A proporção de crianças sem anemia que apresentaram melhores conceitos escolares e que pertencem aos níveis socioeconômicos mais altos foi maior do que a de crianças com anemia, indicando uma maior prevalência de anemia nas crianças que apresentam piores níveis socioeconômicos e rendimento escolar. Contudo, não foi encontrada uma associação estatisticamente significativa entre a anemia ferropriva, os fatores socioeconômicos e o rendimento escolar. 


\section{Conflito de Interesses}

Os autores declaram que não há conflito de interesses com relação à publicação deste manuscrito.

\section{REFERÊNCIAS BIBLIOGRÁFICAS}

1- World Health Organization (WHO). Iron deficiency anemia: Assessment, Prevention, and Control - A guide for programme managers. Geneva: WHO; 2001.

2- Silveira SV, Albuquerque LC, Rocha EJM, Vale Martins MC. Fatores de risco associados à anemia ferropriva em crianças de 12 a 36 meses de creches públicas em Fortaleza. Rev Pediatr. 2007; 2: 6- 70 .

3- Muleviciene A, Sestel N, Stankeviciene S, SniukaiteAdner D, Bartkeviciute R, Rascon J, et al. Assessment of risk factors for iron deficiency anemia in infants and young children: a case-control study. Breastfeed Med. 2018; 13: 493-9.

4- World Health Organization (WHO). Nutritional Anaemias: Tools for Effective Prevention and Control. Geneva: World Health Organization; 2017.

5- Osório MM. Fatores determinantes da anemia em crianças. J Pediatr. 2002; 78: 269-78.

6- Coutinho GGPL, Goloni-Bertollo EM, Bertelli ECP. Iron deficiency anemia in children: a challenge for public health and for society. Sao Paulo Med J. 2005; 123: 88-92.

7- Neuman NA, Tanaka OU, Szarfarc SC, Guimarães PRV, Victora CG. Prevalência e fatores de risco para anemia no Sul do Brasil. Rev Saúde Pública. 2000; 34: 36-56.

8- Braga JAP, Vitalle MSS. Deficiência de ferro na criança. Rev Bras Hematol Hemoter. 2010; 2: 3844.

9- Machado EHS, Leone C, Szarfarc SC. Deficiência de ferro e desenvolvimento cognitivo. Rev Bras Cresc e Desenv Hum. 2011; 21: 368-73.

10- Santos JN, Rates SPM, Lemos SMA, Lamounier JA. Anemia em crianças de uma creche pública e as repercussões sobre o desenvolvimento de linguagem. Rev Paul Pediatr. 2009; 1: 67-73.

11- Heijblom GS, Santos LMP. Anemia ferropriva em escolares da primeira série do ensino fundamental da rede pública de educação de uma região de Brasília, DF. Rev Bras Epidemiol. 2007; 10: 258-66.

12- Carvalho MG, Silva MBS. Hematologia Técnicas Laboratoriais e Interpretação. Belo Horizonte, 1988.

13- Associação Brasileira das Empresas de Pesquisa ABEP. Critério Padrão de Classificação Econômica Brasil, 2008.

14- Jordão RE, Bernardi, Filho AAB. Prevalência de anemia ferropriva no Brasil: uma revisão sistemática. Rev Paul Pediatr. 2009; 27: 90-8.
15- Santos LMP. Bibliografia sobre deficiência de micronutrientes no Brasil 1990-2000: Volume 2bAnemia. 2002.

16- Tsuyuoka R, Bailey JW, Guimarães AMAN, Gurgel RQ, Cuevas LE. Anemia and intestinal parasitic infections in primary school students in Aracaju, Sergipe, Brazil. Cad Saúde Pública. 1999; 15: 41321.

17- Turconi SJ, Turconi VL. Anemia ferropriva: incidência em uma população infantil. Pediatr Mod. 1992; 28: $32-8$.

18- Capanema FD, Lamounier JÁ, Norton RC, Jácome AAA, Rodrigues DA, Coutinho RL, et al. Anemia Ferropriva na infância: novas estratégias de prevenção, intervenção e tratamento. Rev Méd Minas Gerais. 2003; 13.Supl 2: 30-4.

19- Moyses MA. Deficiência de ferro e desenvolvimento cognitivo: um estudo experimental em escolares. Pediatria. 1981; 3: 217-25.

20- Low M, Farrell A, Biggs BA, Pasricha SR. Effects of daily iron supplementation in primary-school-aged children: systematic review and meta-analysis of randomized controlled trials. CMAJ. 2013; 185: E791-802.

21- Santos I, César JA, Minten G, Valle N, Neumann NA, Cercato E. Prevalência e fatores associados à ocorrência de anemia entre menores de seis anos de idade em Pelotas, RS. Rev Bras Epidemiol. 2004; 7: $403-15$

22- Martins IS, Alvarenga AT, Siqueira AAF, Szarfarc SC, Lima F. As determinações biológicas e sociais da doença: um estudo da anemia ferropriva. Rev Saude Publica. 1987; 21: 73-89.

23- Beinner MA, Morais EAH, Filho JDL, Jansen AK, Oliveira SR, Reis IA, et al. Fatores associados à anemia em adolescentes escolares do sexo feminino. Rev B Saud Publ. 2013; 37: 439-51.

24- Rocha DS, Capanema FD, Netto MP, Franceschini SCC, Lamounier JA. Prevalência e fatores determinantes da anemia em crianças assistidas em creches de Belo Horizonte - MG. Rev Bras Epidemiol. 2012; 15: 675-84.

25- Silva LSM, Giugliani ERJ, Aerts DRGC. Prevalência e determinantes de anemia em crianças de Porto Alegre, RS, Brasil. Rev Saude Publica 2001; 35: 6673.

26- Pessoa MC, Jansen AK, Melendez GV, Lopes JD, Beinner MA. Anemia em crianças e fatores associados em região urbana. Rev Min Enferm. 2011; 15: 54-61.

27- Oliveira CSM, Cardoso MA, Araújo TS, Muniz PT. Anemia em crianças de 6 a 59 meses e fatores associados no Município de Jordão, Estado do Acre, Brasil. Cad Saúde Pública. 2011; 27: 1008-20.

28- Ferraz ST. Anemia Ferropriva na Infância: uma Revisão para Profissionais da Atenção Básica. Rev APS. 2011; 14: 101-10. 